\title{
Sustainable mobile computing
}

\author{
Yuhang Yang • Maode Ma
}

Received: 4 February 2013 / Accepted: 7 February 2013 / Published online: 9 August 2013

(C) Springer-Verlag Wien 2013

Mobile computing has the potential to enable a large class of applications, such as smart phone, mobile internet, mobile game and mobile multimedia, etc. However, as a high-tech, it is also a high-energy-consuming one. Sustainable mobile computing with the aiming of energy costs and $\mathrm{CO}_{2}$ emissions' reducing, and environment protecting, has become a potential research focus for all the next-generation communications' and networks' designers. In spite of the increasing demand for all kinds of mobile computing services, we are still lacking a clear understanding of sustainable mobile computing and techniques, as well as best practices, to design energy-efficient, utility, pervasive, scalable, autonomy-oriented mobile computing and applications. The objective of this special issue is to bring together state-of-the-art research contributions that address these key aspects of sustainability in mobile computing and applications.

There were nine papers submitted for consideration for publication in this special issue. After two rounds of rigorous peer-review and revision processes, only three papers were selected for publication. Unfortunately not all of the excellent papers can be selected into this special issue due to limited space available. And we believe that these papers included in this special issue represent the outstanding quality of the research outcomes in this field.

The first paper, "Energy-efficient Job Stealing for CPU-intensive processing in Mobile Devices", by Juan Manuel Rodriguez, Cristian Mateos, and Alejandro Zunino, explores an analysis of different schedulers based on job stealing for mobile

\footnotetext{
Y. Yang $(\varangle)$

Department of Electronic Engineering, Shanghai Jiao Tong University,

Shanghai 200240, China

e-mail: yhyangsjtu@gmail.com

M. Ma

School of Electrical and Electronic Engineering, Nanyang Technological University,

Nanyang Avenue, Singapore, Singapore
} 
computational grids, which have been designed to consider energy consumption and battery status for mobile devices.

The second paper, "An Efficient Geographic Routing Protocol Design in Vehicular Ad-Hoc Network”, by Jian-Ming Chang, Han-Chieh Chao, Chin-Feng Lai, and Rongbo Zhu, presents a novel power-efficient geographic routing algorithm that uses the direction, density and distance between nodes in the crossroad routing strategy, to improve the link stability of vehicular adhoc networks in mobile environment.

The last paper, "Energy Efficient Smart-Relay-Based Cooperative MAC for Wireless Networks", by Mohammad Helal Uddin Ahmed, Choong Seon Hong, and Sungwon Lee, proposes an energy efficient smart-relay-based cooperative MAC protocol to optimize the impacts of hidden and exposed terminal problems, and an efficient relay selection mechanism is also illustrated to consider a back-up relay to increase the reliability of the transmission in wireless networks.

The Guest Editors would like to thank all the authors for their contributions. And special thanks go to all reviewers for their great effort, timely responses and constructive comments and suggestions. Thanks also go to Prof. Schahram Dustdar, the Editor-in-Chief of Computing Journal, and the journal editorial staff who helped us through out the entire process. 